\title{
Educación para la salud de las mamas de mujeres ancianas a través de círculos de cultura
}

\author{
Health education of elderly women's breasts by \\ circles of culture
}

\section{Educação para saúde das mamas de mulheres idosas através de círculos de cultura}

\author{
Államy Danilo Moura e Silva ${ }^{1}$, Izabel Cristina Falcão Juvenal \\ Barbosa $^{2}$, Inez Sampaio Nery ${ }^{3}$, Nayra Samanta Alves Luz ${ }^{4}$, Ana \\ Fátima Carvalho Fernandes ${ }^{5}$
}

\begin{abstract}
${ }^{1}$ Enfermeiro. Mestre em Enfermagem pela Universidade Federal do Piauí. Teresina, Piauí, Brasil. Correo electrónico: allamydanilo@hotmail.com

${ }^{2}$ Enfermeira. Doutora em Enfermagem pela Universidade Federal do Ceará. Professora do Curso de Enfermagem da Universidade Federal do Piauí. Floriano, Piauí, Brasil. Correo electrónico: izabelbarbosa@ufpi.edu.br
\end{abstract}

\begin{abstract}
${ }^{3}$ Enfermeira. Doutora em Enfermagem pela Universidade Federal do Rio de Janeiro. Professora de PósGraduação em Enfermagem da Universidade Federal do Piauí. Teresina, Piauí, Brasil. Correo electrónico: inezsampaionery11@gmail.com

${ }^{4}$ Enfermeira. Graduada em Enfermagem pela Universidade Federal do Piauí. Floriano, Piauí, Brasil. Correo electrónico: nayrasamanta_21@hotmail.com

${ }^{5}$ Enfermeira. Doutora em Enfermagem pela Universidade Federal de São Paulo. Professora de Pós-Graduação em Enfermagem da Universidade Federal do Ceará. Fortaleza, Ceará, Brasil. Correo electrónico: afcana@ufc.br

Cómo citar este artículo en edición digital: Silva, A.D.M., Barbosa, I. C.F.J., Nery, I.S., Luz, N.S.A. E Fernandes, A.F.C. (2019). Educación para la salud de las mamas de mujeres ancianas a través de círculos de cultura. Cultura de los Cuidados (Edición digital), 23(53). Recuperado de http://dx.doi.org/10.14198/cuid.2019.53.31
\end{abstract}

Correspondencia: Államy Danilo Moura e Silva Universidade Federal do Piauí. Campus Ministro Petrônio Portella. Departamento de Enfermagem. Teresina, Piauí, Brasil

Correo electrónico: allamydanilo@hotmail.com Recibido: 08/11/2018; Aceptado: 22/01/2019

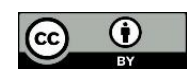

\section{ABSTRACT}

Introduction: Health education as the health promotion strategy should be a social practice focused on the daily questioning, appreciation of life experiences, adapted to different realities.

Objective: The study has the general objective to describe the development of educational activities for prevention and early detection of breast cancer in older women through crop circles.

Methods: This is an exploratory descriptive research with a qualitative approach. Subjects were 13 elderly women enrolled in a Basic Health Unit in the city of Floriano, Piauí, Brazil. The theoretical foundation of culture circles created by Paulo Freire, and the lines evaluated by 
Bardin content analysis.

Results: The crop circles were a dialogue enhancement of the environment in which the words were generated consonant with the experiences and living habits of the participants, encouraging interaction. Speaking at breast cancer, the words expressed were related to fear and despair, confirming the association of this neoplasm with feelings of intense sadness.

Conclusion: We conclude that the implementation of crop circles led to the participant group, the reflectivity of their conduct to position themselves as protagonists of their history of health/illness related to what was put to discussion, facilitating the achievement of a better quality of life.

Keywords: Breast neoplasms, health promotion, health education.

\section{RESUMO}

Introdução: A educação em saúde como estratégia da Promoção da saúde, deve ser uma prática social focada na problematização do cotidiano, valorização das experiências de vida, adaptada às diferentes realidades.

Objetivo: O estudo tem como objetivo geral descrever o desenvolvimento de ações educativas para prevenção e detecção precoce do câncer de mama em mulheres idosas através de círculos de cultura.

Métodos: Trata-se de uma pesquisa descritiva exploratória, com abordagem qualitativa. Participaram 13 mulheres idosas cadastradas em uma Unidade Básica de Saúde do município de Floriano, Piauí, Brasil. A fundamentação teórica foram os círculos de cultura criados por Paulo Freire, sendo as falas avaliadas por meio da análise de conteúdo de Bardin.

Resultados: Os círculos de cultura constituíram um ambiente de valorização do diálogo em que as palavras geradas eram consoantes com as vivências e os hábitos de vida das participantes, favorecendo a interação. Ao falar em câncer de mama, as palavras expressadas estavam relacionadas a medo e desespero, confirmando a associação dessa neoplasia com sentimentos de intensa tristeza.

Conclusão: Conclui-se que a implementação dos círculos de cultura propiciou a capacidade de reflexão de suas condutas ao posicionarem-se como protagonistas de sua história de saúde/doença relacionada ao que era posto a discussão, facilitando o alcance de uma melhor qualidade de vida.

Palavras chave: Neoplasias da mama, promoção da saúde, educação em saúde.

\section{RESUMEN}

Introducción: La educación en salud como estrategia de la Promoción de la salud, debe ser una práctica social enfocada en la problematización del cotidiano, valorización de las experiencias de vida, adaptada a las diferentes realidades.

Objetivo: El objetivo general del estudio fue describir el desarrollo de acciones educativas para prevención y detección precoz del cáncer de mama en mujeres ancianas a través de círculos de cultura.

Métodos: Se trata de una investigación descriptiva exploratoria, con enfoque cualitativo. Participaron 13 mujeres ancianas registradas en una Unidad Básica de Salud del municipio de Floriano, Piauí, Brasil. Los fundamentos teóricos fueron los círculos de cultura creados por Paulo Freire, siendo que los discursos fueron evaluados por medio del análisis de contenido de Bardin.

Resultados: Los círculos de cultura 
constituyeron un ambiente de valorización del diálogo en que las palabras generadas eran consonantes con las vivencias y los hábitos de vida de las participantes, favoreciendo la interacción. Al hablar en cáncer de mama, las palabras expresadas estaban relacionadas al miedo y desespero, confirmando la asociación de esa neoplasia con sentimientos de intensa tristeza.

Conclusión: Se concluye que la implementación de los círculos de cultura fomentó la capacidad de reflexión de sus conductas al posicionarse como protagonistas de su historia de salud/enfermedad relacionada a lo que era puesto en discusión, facilitando el alcance de una mejor calidad de vida.

Palabras clave: Neoplasias de mama, promoción de la salud, educación en salud.

\section{INTRODUÇÃO}

O significado pertinente para Promoção da Saúde ganhou atenção mais intensa a partir da segunda metade do século passado, sendo pauta de discursões e apresentada como um plano a nível mundial que visa diminuir os vários e recorrentes problemas de saúde que afetam a população, por meio da educação em saúde (Janini, Bessler \& Vargas 2015; Pinheiro et al., 2015). A educação em saúde como estratégia de Promoção da Saúde, deve ser uma prática social focada na problematização do cotidiano, valorização das experiências de vida, adaptada às diferentes realidades. Sendo uma prática social, a educação em saúde traz consigo uma visão cultural, definida pelos valores, crenças e as formas como as pessoas vivem e veem o mundo (Alves \& Aerts, 2011). Dentre os métodos de educação em saúde, o Círculo de Cultura criado por Paulo Freire, é um ambiente onde todos têm a palavra, leem e escrevem o mundo, constituindo um espaço de trabalho, pesquisa, ações, dinâmicas e vivências que possibilitam a construção coletiva do conhecimento (Monteiro \& Vieira, 2010).

De forma explicativa, considera-se "Círculo" porque todos estão em volta de uma equipe de trabalho, norteados por um animador de debates que participa das atividades comuns em que todos aprendem e ensinam simultaneamente, tendo como objetivo principal, a participação ativa dialogada dos participantes. "De Cultura", porque os círculos vão além da aprendizagem individual, produzindo modos inovadores, solidários e coletivos de pensar (Brandão, 2005). Com isso, este estudo justifica-se pelo pressuposto de que ações educativas devem fazer parte das ações em enfermagem, no papel de facilitador da aquisição de conhecimento para a prevenção de problemas de saúde pública difíceis de obter controle, como é o caso do câncer de mama, que apesar do incremento de novas tecnologias para o diagnóstico e da criação de novas políticas por parte das autoridades, observa-se uma elevada taxa de morbimortalidade.

O câncer de mama é um grave problema de saúde pública, devido à dificuldade de prevenção primária, que é a forma de eliminar os fatores de risco ou diagnosticar e tratar precocemente a neoplasia, constituindo-se como o tipo de câncer mais prevalente nas mulheres. A sua incidência está relacionada com a idade avançada, embora constitua uma neoplasia relativamente frequente em mulheres mais jovens (Checka et al., 2012; Almeida \& Zeferino, 2013; Fernandes et al., 2012). Ainda que se tenha aumentado as ações em saúde do idoso nos últimos anos, o maior enfoque na promoção da saúde para essa faixa etária 
não acompanha o ritmo de crescimento populacional dos idosos no mundo, onde esse fenômeno ocorre de forma radical e acelerada Eliopoulos, 2011; Veras, 2009). Uma forma de melhorar a atenção ao idoso é fazendo-o com que crie um vínculo com o sistema de saúde, sendo a Unidade Básica de Saúde, um espaço privilegiado para atenção integral à saúde, já que sua proximidade com a comunidade possibilita atuar de forma contextualizada na realidade vivenciada pelo idoso, favorecendo a promoção da saúde (Gautério et al., 2013).

Desse modo, fazemos o seguinte questionamento: Os círculos de cultura se constituem como estratégia eficaz na promoção de ações educativas em saúde mamária para mulheres idosas? Entende-se a necessidade de intensificar as ações de promoção da saúde da mulher idosa por meio de metodologias participativas, quanto às formas de detectar rápido o câncer de mama, diante de suas proporções de mortalidade. Ao considerar as características do envelhecimento e a necessidade de intensificar e criar novos meios para promover saúde da idosa no âmbito do câncer de mama, esse trabalho tem como objetivo descrever o desenvolvimento de ações educativas para prevenção e detecção precoce do câncer de mama em mulheres idosas através de círculos de cultura.

\section{MÉTODOS}

Trata-se de uma pesquisa descritiva, com abordagem qualitativa, tendo como método a pesquisa ação, desenvolvida nos meses de outubro e novembro de 2015, numa Unidade Básica de Saúde do município de Floriano, Piauí, nordeste do Brasil. A amostra foi composta por treze mulheres idosas que atenderam aos seguintes critérios de inclusão: ter idade igual ou superior a 60 anos, ser residente da área da Unidade Básica de Saúde onde a pesquisa foi executada, aceitar participar do estudo de forma voluntária após esclarecimentos dos objetivos da pesquisa e assinar termo de consentimento. Não fizeram parte da amostra, as idosas que possuíssem diagnóstico de doença degenerativa neurológica, que impossibilitasse a tomada de decisões.

Para a coleta dos dados foram realizadas atividades educativas baseadas na fundamentação teórica dos círculos de cultura criado por Paulo Freire e entrevista semiestruturada. Foram iniciadas as atividades com o total de quatro círculos de cultura realizados em duas sextas feiras. Cada sexta aconteciam dois círculos com duração de uma hora e um intervalo com lanche e música dividindo-os. No. 1. ${ }^{\mathrm{o}}$ encontro: dinâmica de apresentação e discussão acerca do conceito de câncer instigando a geração de palavras. A partir das colocações das participantes, o facilitador abordou o conceito de câncer de uma forma clara e correlacionada com o cotidiano dos sujeitos envolvidos.

No. 2. ํ encontro: Diálogo sobre os métodos de autocuidado com suas mamas a partir do autoexame, geração de ideias sobre a temática detecção precoce, exame clínico das mamas e mamografia. 3. o encontro: Leitura do conto, uma história fictícia narrada pelo animador para instigar o raciocínio crítico das participantes, com base no conhecimento adquirido, e testar a tomada de decisão frente ao aparecimento de quaisquer lesões sugestivas do câncer de mama. 4. ${ }^{\circ}$ encontro: Dinâmica de "palavras articuladoras de pensamento crítico" (Brandão, 2005). As palavras a serem propostas: idosa, saúde, amor, família e paz dentre outras, escritas em cartazes e 
possuíam um desenho representativo, estimulando o diálogo entre todas as participantes.

Para a análise dos dados as informações organizadas através dos relatos transcritos na íntegra, analisados na ordem da convergência das falas, e em seguida agrupados em categorias semelhantes de análise de conteúdo conforme preconiza o referencial metodológico de Bardin, que utiliza procedimentos sistemáticos e objetivos de descrição de mensagens. Condiz uma organização cronológica para as diferentes fases da análise de conteúdo, constituindo-se como: 1- pré-análise; 2exploração do material e 3- tratamento dos resultados, inferência e a interpretação (Bardin, 2004).

Na fase 1, denominada de pré-análise, foi realizada a organização, caracterizando-se por um período de intuições que tem por objetivo sistematizar as ideias iniciais de forma a conduzir um plano de análises (Bardin, 2004). Nesta fase foram escolhidas as falas e formulada as hipóteses.

A exploração do material, compreende a fase 2, considerada longa e exaustiva por consistir na codificação ou enumeração de regras previamente formuladas. Foram feitas transformações dos dados brutos do texto, através de recorte, enumeração e classificação com o intuito de atingir uma compreensão do texto (Bardin, 2004).

$\mathrm{Na}$ fase 3, tratamento dos resultados, inferência e interpretação, os resultados brutos receberam um tratamento para tornarem-se válidos e significativos (Bardin, 2004). De posse destes resultados, foram realizadas as inferências e interpretações dos objetivos traçados e correlacionados à literatura pertinente.

Para manter o anonimato, as participantes foram codificadas com as siglas (P1, P2, P3... P13). Esta pesquisa faz parte do macroprojeto intitulado "Um toque pela vida" que tem por objetivo desenvolver ações de educação e promoção da saúde para mulheres do referido município com enfoque na prevenção e detecção precoce do câncer de mama, que foi submetido e aprovado pelo Comitê de Ética de Pesquisa da Universidade Federal do Piauí sob o parecer: $\quad$ 0214.0.045.000-11. Buscou-se atender as diretrizes da Resolução 466/12 do Conselho Nacional de Saúde do Ministério da Saúde do Brasil, a qual se refere às pesquisas com seres humanos e destaca a proteção ao bem estar dos indivíduos pesquisados, bem como o respeito aos valores culturais, morais, religiosos e éticos.

\section{RESULTADOS}

Ao iniciarem as ações, realizou-se a dinâmica dos "pares de rosas" onde disponibilizou-se um buquê com rosas em pares de cores distintas. As idosas que pegassem as rosas correspondentes ao par deveriam conversar, depois apresentar uma a outra ao grupo e ainda complementar a fala com suas expectativas quanto aos encontros. A dinâmica de apresentação serviu para familiarizar os participantes do círculo e tornar um ambiente relacional.

\section{Palavras geradoras por meio das definições do câncer de mama}

De acordo com a proposta dos círculos de cultura, nesse primeiro momento as participantes foram estimuladas ao diálogo e geração de palavras que norteassem o debate, a partir do questionamento do que significava o câncer para elas:

Tenho muitas dúvidas sobre câncer, porque quando se ver, de repente a doença aparece, como na minha vizinha (P1).

Ouvi dizer que é perigoso e mata ligeiro (P8). 
Já eu, tenho horror! Eu não gosto nem de falar no câncer, eu digo é: aquela doença feia (P3).

Percebemos, diante das falas, uma forte associação do câncer como uma doença muito ruim, talvez como uma das piores na percepção das mulheres, quando referem palavras como medo, horror e angústia que vêm relacionadas às dúvidas também relatadas. Com isso, iniciaram-se os procedimentos de educação em saúde propriamente ditos, conceituando o câncer de mama de uma forma leve e criativa comparando-o com um caranguejo, diante de suas características de esconder-se e por possuir patas espalhadas com poder de fixação, além de sua agressividade e imprevisibilidade:

Do mesmo jeito é um carrapato quando gruda nos bichos, e ainda se escondem. Se a gente demorar para ver, fica empesteado (P9).

A fala da P9 ao fazer analogia com algo mais próximo da realidade local, reflete alguns pressupostos da educação popular segundo Freire (2007) em que esta se fundamenta na criatividade e estimula a reflexão e ação das pessoas sobre sua realidade, onde o facilitador precisa perceber-se como aprendiz, diante de um ambiente cultural particular, buscando adotar o caráter e as necessidades sociais e culturais dos participantes, no processo educativo.

Percebeu-se a repetição das palavras "medo" e outras que denotam sentimentos de estigma, que se intensificaram com algumas informações epidemiológicas sobre o câncer de mama que foram colocadas ao introduzir essa parte da conversa:

Me dar medo também... Ainda mais para mim que tive gente minha doente com isso (P10).

O câncer nas mamas é o que dá mais medo, porque parece que é mais perigoso e que mata mais, passou até no jornal (P7).

Falas como essas supracitadas ocasionou um ambiente de valorização da escuta e eufemismo por parte do animador, quanto às informações explanadas, com intuito de não causar sensacionalismo e aumentar as preocupações com o câncer de mama, a partir dos dados negativos narrados. Nesse contexto, para diminuir a tensão e tranquilizar as participantes, objetivou-se então gerar pensamentos positivos a partir de uma boa notícia norteadora: Quanto mais cedo o câncer de mama for descoberto, maiores são as chances de cura. E o que fazer para prevenir problemas maiores?

Tem a mamografia, que é um exame que aperta. Eu sei que é muito importante esse exame, mas não faço há um tempo (P2).

Hoje em dia as coisas da medicina estão melhores, tem tecnologia. Antes, as pessoas adoeciam e não se tratavam (P2).

As colocações da P2, apesar de empíricas, são bastante pertinentes, por trazerem questões reais dos avanços das tecnologias duras para diagnóstico precoce do câncer de mama e dos tratamentos para a doença, tais informações que foram reforçadas pelo animador e que geraram pensamentos positivos, voltando ao equilíbrio e estimulando novos questionamentos de outras maneiras de prevenir e detectar precocemente um câncer.

\section{Detecção precoce do câncer de mama}

No segundo círculo de cultura, iniciaramse as atividades ao estimular o diálogo do que foi debatido anteriormente a respeito das estratégias para a detecção precoce do câncer de mama. A princípio, buscou-se enfocar o autocuidado a partir dos questionamentos: como descobrir cedo um câncer de mama? Você conhece seu corpo? 
A introdução do debate teve o intuito de que as mulheres refletissem sobre o corpo normal e o corpo com alterações, em que para se descobrir possíveis alterações corporais é necessário conhecê-lo. Para ser menos invasivo ao falar de toque e de corpo com as idosas, comparou-se essa ideia de descobrir algo diferente no que costuma estar normal, como na própria casa das participantes:

Na minha casa, nas minhas coisas, eu sei quando está faltando alguma coisa e quando está alguma coisa diferente (P1).

Com base no que P1 referiu, o animador fez analogia do conhecimento dos objetos de casa com o conhecimento do corpo, especialmente das mamas quanto à descoberta de alterações mamárias. Nesse sentido, as mulheres foram indagadas quanto ao interesse em aprender as técnicas para o autoexame das mamas. Com resposta positiva, iniciaram-se as demonstrações em que todas participaram.

Além da demonstração da palpação, esclareceu-se quanto à inspeção e outras informações pertinentes como: ressaltar que o autoexame das mamas é um método complementar e não deve substituir o exame clínico e a mamografia para o rastreamento e detecção precoce de possíveis neoplasias mamárias:

Quando eu estiver no banho, vou lembrar de fazer esse exame (P12).

Eu pensei que fosse mais difícil. Gostei mais dessa parte deitada na cama, e se Deus me livre encontrar o caroço eu corro no médico (P5).

Complementando essa atividade, foram entregues às participantes algumas próteses mamárias realísticas de silicone em que algumas delas possuíam nódulos palpáveis que mimetizavam um câncer de mama. À medida que identificavam os tumores nas próteses, percebiam-se as reações de espanto, receio ao tocar e a curiosidade de algumas delas.

\section{Reflexão a partir de narrativa}

No terceiro encontro, as idosas escutaram e participaram da leitura de um conto, uma história fictícia elaborada pelo animador do grupo, onde uma personagem idosa encontra alterações mamárias. A dinâmica visou instigar o raciocínio crítico das participantes, com base no conhecimento já adquirido, quanto à tomada de decisão frente ao aparecimento de quaisquer umas das lesões sugestivas de câncer de mama. As participantes ficaram empolgadas e até emocionadas com a história. A partir daí, a diálogo foi aberto visando responder ao questionamento:

(Suspiro) ... eu acho que ia ficar desesperada que nem ela. Não, não, eu nem sei o quê eu ia fazer (P7).

Ela tem que fazer o quê vocês disseram na sexta, tem que ir procurar tratamento (P2).

Eu primeiro ia orar a Deus, para me dar uma luz. Deus é poderoso, ele sabe o melhor para nós todos (P9).

Sequencialmente, foi reforçada a importância de consulta ao profissional da saúde, diante do aparecimento de alterações mamárias, esclarecendo rapidamente quanto ao sistema de referências na saúde.

\section{Palavras articuladoras do pensamento crítico}

O quarto círculo de cultura trouxe as "palavras articuladoras de pensamento crítico" que somam às palavras geradoras para provocar um pensamento crítico mais demorado e articulação com as vivências e os sentimentos das participantes do círculo (Freire, 2007).

Utilizaram-se palavras como idosa, saúde, 
amor, família e paz, que foram expostas em cartazes com desenhos representativos ao lado auxiliando as idosas que não sabiam ler:

Ser idosa, por mais que apareçam muitas doenças... (risos)... é um dádiva de Deus (P13).

O que seria da gente sem a família. Quando eu vejo minha família feliz, esqueço até das coisas ruins. Por isso família é amor, família é tudo (P4).

A saúde é o que me dar coragem, sem saúde eu não estaria aqui com vocês ouvindo essas coisas importantes (P11).

As idosas destacaram a importância do "ser idosa e ter família", como continuidade das gerações e demonstrações de afeto, sendo considerados essenciais em possíveis processos patológicos, considerados preocupantes pelos idosos, conforme as falas sobre saúde, em que a falta desta, sugere improdutividade e restrições.

\section{DISCUSSÃO}

Os sentimentos de medo ao falar em câncer de mama, transparecem ser uma ameaça à vida e à integridade física da mulher, que ao pensar na possibilidade de diagnóstico, vivencia a expectativa de um futuro incerto, preocupando-se com as dificuldades, que vem acompanhada do medo da morte e mutilação (Ramos, 2012).

No presente estudo, observamos a carência de conhecimento das participantes quanto ao câncer de mama, acompanhado de medos e tabus. Com isso, o processo de educação em saúde foi embasado no conhecimento prévio, fazendo associações com a realidade e a cultura local.

É importante trabalhar o dialeto e vivências, pois ao lidar com pessoas, a cultura mostra a visão do mundo dos indivíduos, sendo necessário seu entendimento para que o educador possa buscar intervenções consoantes com a realidade dos educandos (Martins et al., 2013; Beserra et al., 2011).

Quanto às medidas de detecção precoce, observamos pouco conhecimento e omissão das mulheres ao tocarem o próprio corpo. $\mathrm{O}$ nível de conhecimento baixo sobre a temática é frequente na população idosa, que apesar da alta incidência, são elas que em menor quantidade de vezes fazem mamografias, são submetidas a exames clínicos por profissionais de saúde e realizam o autoexame, deixando-as mais exposta aos riscos (Almeida, Guerra \& Filgueiras, 2012). Com isso, é necessário implementar estratégias de educação em saúde mamária, já que o conhecimento das mulheres sobre detecção do câncer ainda é insuficiente (Fernandes et al., 2012).

A narrativa fictícia permitiu a reflexão do grupo. As palavras referenciadas sugerem a uma antecipação de sentimentos das mulheres idosas quanto ao possível diagnóstico de câncer de mama, considerando os preceitos de Kubler-Ross (1997) ao estudar as fases do adoecer e morrer, observa-se forte espiritualidade. Um suposto diagnóstico de câncer confronta o sujeito com a questão do imprevisível, da finitude e da morte, e por ser uma doença potencialmente letal, traz a ideia de perda do corpo saudável, a perda da sensação de invulnerabilidade e a perda do domínio sobre a própria vida.

A provocação das palavras articuladoras do pensamento crítico foi relevante para a consciência crítica das situações cotidianas, ao causar entusiasmo nas formas de pensar e enfocar assuntos pouco expressados, renovando as maneiras de pensá-los.

Essas palavras junto às tantas outras referidas durante os encontros anteriores, pretenderam inteirar-se com o que é 
cotidiano e o que deve ser bom para as idosas, causando um momento de reflexãoação "como prática da liberdade, um ato de conhecimento, uma aproximação crítica da realidade" (Freire, 2005).

As palavras expressadas a partir daquelas expostas em cartazes retratam um processo de internalização/externalização de sentimentos através, também dos signos e símbolos que estimulam o imaginário (Freire, 2005). O falar sobre ser idosa ofereceu a reflexão sobre o estar no mundo e na sociedade, reconhecendo que apesar da idade avançar, não devemos percebê-la como algo ruim que vem acompanhado de coisas ruins, como as doenças, mas sim como motivo de altivez.

\section{CONCLUSÃO}

$\mathrm{O}$ ambiente de encontro e descoberta proposto pelo método de Paulo Freire implementado neste estudo, contribuiu para o descobrimento de potencialidades não conhecidas pelas próprias mulheres idosas, muitas vezes marginalizadas por situações socioculturais. Esse espaço descontraído, em formato de círculo, norteado por questionamentos e planejado com criatividade, causou a geração de sentimentos compartilhados onde a troca de experiência era mútua, favorecendo a aprendizagem coletiva a partir da junção do saber científico debatido de forma dinâmica e adaptado à realidade das participantes.

Reconhece-se que os círculos de cultura se constituem como estratégia efetiva para a promoção da saúde de idosas quanto ao câncer de mama, pois propiciou ao grupo participante a capacidade de reflexão de suas condutas ao posicionarem-se como protagonistas de sua história de saúde e doença relacionada ao que era posto a discussão, facilitando o alcance de uma melhor qualidade de vida.

Sugere-se a divulgação dos círculos de cultura no meio científico no âmbito da saúde e da enfermagem, como estratégia educativa a ser adotada pelos profissionais da área, diante de seu forte embasamento teórico-prático elaborado por Paulo Freire e por sua comprovada eficácia confirmada neste estudo e em outras literaturas que versavam educação em saúde na comunidade.

\section{REFERÊNCIAS BIBLIOGRÁFICAS}

- Almeida, O.J.A. \& Zeferino, L.C. (2013). Rastreamento do câncer de mama na mulher idosa. Revista Brasileira de Cancerologia, 59(4), 555-557.

- Almeida, T.R., Guerra, M.R. \& Filgueiras, M.S.T. (2012). Repercussões do câncer de mama na imagem corporal da mulher: um revisão sistemática. Physis Revista de Saúde Coletiva. 22(3),1003-1029. Recuperado de: http://www.scielo.br/pdf/physis/v22n3/09.pdf.

- Alves, G.G. \& Aerts, D. (2011). As práticas de educação em saúde e a Estratégia Saúde da Família. Ciência e Saúde Coletiva, 16(1), 319-125.

- Bardin, L. (2004). Análise de Conteúdo. 3 ed. Lisboa: Persona.

- Beserra, E.P., Torres, C.A., Pinheiro, P.N.C., Alves, M.D.S. \& Barroso, M.G.T. (2011). Pedagogia freireana como método de prevenção de doenças. Ciência \& Saúde Coletiva,16(1), 1563-1570. Recuperado de: http://www.scielo.br/scielo.php?pid=S1413$81232011000700092 \&$ script=sci_abstract\&tlng $=$ pt.

- Brandão, C.R. (2005). O que é o método Paulo Freire. 7. ${ }^{a}$ ed. São Paulo: Brasiliense.

- Checka, C.M., Chun, J.E., Schnabel. F.R., Lee, J., y Toth, H. (2012). The relationship of mammographic density and age: implications for breast cancer screening. Am J Roentgenol, 198(3), 292-295.

- Eliopoulos, C. (2011). Enfermagem gerontológica. Porto Alegre: Artmed.

- Fernandes, A.F.C., Bonfim, I.M., Araújo, I.M.A., Silva, R.M., Barbosa, I.C.F.J. \& Santos, M.C.L. (2012). Significado do cuidado familiar à mulher mastectomizada. Esc Anna Nery, 16(1), 27-33. Recuperado

de: http://www.scielo.br/pdf/ean/v16n1/v16n1a04.pdf. - Freire, P. (2005). Pedagogia da autonomia: saberes 
necessário à prática educativa. 31. a ed. Rio de Janeiro: Paz e Terra.

- Freire, P. (2007). Educação como prática da liberdade. Rio de Janeiro: Paz e Terra.

- Gautério, D.P., Vidal, D.A.S., Barlem, J.G.T., y Santos, S.S.C. (2013). Ações educativas do enfermeiro para a pessoa idosa: estratégia saúde da família. Rev. enferm. UERJ, 21(2), 824-828.

- Janini, J.P., Bessler, D. \& Vargas, A.B. (2015). Health education and health promotion: impact on quality of life of elderly. Saúde Debate, 39(105), 480-490.

- Kubler-Ross, E. (1997) The wheel of life: a memoir of living and dying. New York: Touchstone.

- Martins, A.K.L.M., Barbosa, I.C.J.F., Silva, A.D.M., Borges, B.V.S., y Santos, L.R. (2013). O paradigma manicomial em tela: relato de experiência de acadêmicos de enfermagem. Sanare,12(2), 54-58.

- Monteiro, E.M.L.M. \& Vieira, N.F.C. (2010) Educação em saúde em círculos de cultura. Revista Brasileira de Enfermagem, Brasília, 63(3), 397-403.

- Pinheiro, D.G.M., Scabar, T.G., Maeda, S.T., Fracolli, L.A., Pelicioni, M.C.F. \& Chiesa, A.M. (2015). Competências em promoção da saúde: desafios da formação, Saúde Soc., 24(1), 180-188.

- Ramos, W.S.R. (2012). Sentimentos vivenciados por mulheres acometidas por câncer de mama. J Health Sci Inst., 30(3), 241-248.

Veras, R. (2009). Envelhecimento populacional contemporâneo: demandas, desafios e inovações. Rev Saúde Pública, 43(3), 548-5542. 